\section{Aberrant Sectoral Biliary Duct Encountered Retrospectively on MRCP with Postoperative Collection}

Sir,

Right posterior sectoral duct (RPSD) joining cystic duct is an extremely rare intra-hepatic biliary variant in the normal population. A 35-year lady during MRCP, presented to Dow University of Health Sciences (DUHS), Pakistan with the symptoms of obstructive jaundice, dyspepsia, nausea, and vomiting after laparoscopic cholecystectomy. The serum laboratory tests revealed raised alkaline phosphatase, gamma glutamyl transferase levels, and $3.0 \mathrm{mg} / \mathrm{dl}$ of direct bilirubin. The suspicion was slipped residual gall stone obstructing common bile duct(CBD); a notherpossibility was of biliary stricture. The MRCP acquisition showed drainagetubetip seen in the sub-hepatic region with minimal peri-hepatic free fluid. There was significant dilatation of the CBD, $(1.2 \mathrm{~cm}$ in diameter), and of biliary radicals. Abrupt narrowing was identified in mid-CBD. About $1 \mathrm{~cm}$ of mid CBD was not visualised. Distal CBD appeared normal in caliber. The possibility of a short segment biliary stricture, likely ischemic in etiology, due to excessive cauterisation in the surgical field was considered. A cystic area was identified in the region of gallbladder fossa, measuring approximately. On close look, it was noted that segment V and VI bile radicals were draining into anomalous RPSD communicating with that "postoperative collection" (Figures 1 and 2), which was actually a cystic duct stump. The patient subsequently underwent percutaneous trans-hepatic cholangiography (PTC) for external drainage of the biliary system and confirmation of findings. There was non-visualisation of RPSD. Unfortunately, in this patient, the aberrant RPSD cannot be salvaged, as its distal end was cauterised, so operative collection was drained and Roux-en-y hepaticojejunostomy was done. The patient made an uneventful recovery. As there was CBD injury, the RSPD cannot be anastomosed to the main bile duct. The dismal outcome would be the outcome of segment $\mathrm{V}$ and VI hepatic atrophy due to sectoral bile duct obstruction.

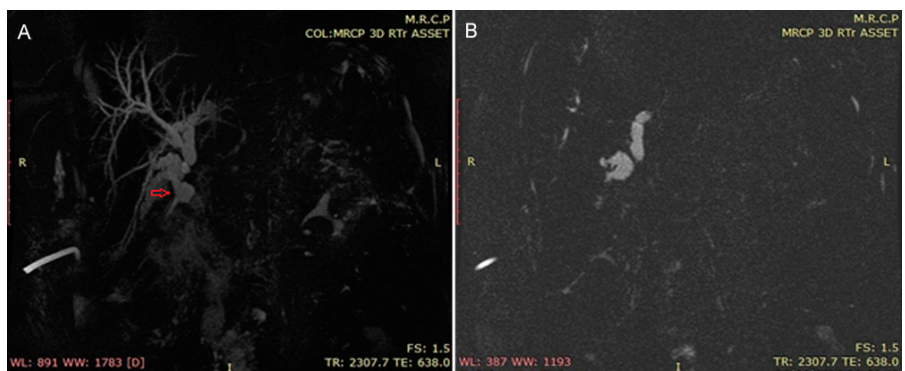

Figure 1: MRCP showing dilated right posterior sectoral duct (RPSD) draining into the dilated non-communicating cystic duct stump.

Biliary duct injury (BDI), being a common complication in laparoscopic cholecystecystomy, ${ }^{1}$ most probably arose from faulty surgical technique in this case, resulting in biliary perforation. Intra-hepatic bile duct anatomy is complex with many common and uncommon variations. MRCP is a reliable non-invasive imaging method for demonstration of bile ductmorphology, which is useful to plan complex surgeries and prevent iatrogenic injuries. ${ }^{2}$ Sureka et al in 2016 studied biliary anatomical variations. ${ }^{3}$ Total VIII branching patterns of right hepatic duct (RHD) were described. Type I typical: RPSD joining right interior sectoral duct (RASD) medially to form RHD; Type II was trifurcation: Simultaneous emptying of the RASD, RPSD, and left hepatic duct (LHD) into the CHD. Type III was rare, anomalous drainage of RPSD subcategorised as:

RPSD joining LHD; (b) RPSD joining CHD; and (c) RPSD joining cystic duct.
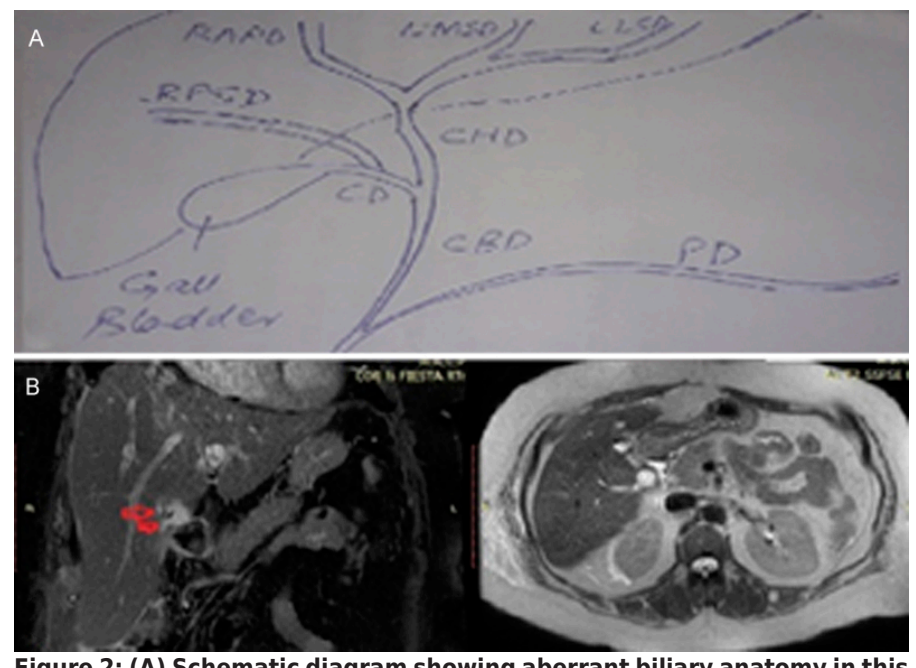

Figure 2: (A) Schematic diagram showing aberrant biliary anatomy in this case. (B) MRCP showing dilated segment $V$ and $V I$ bile radicals draining into anomalous right posterior sectoral duct (RPSD) (red arrows).

In the study of 224 patients by Sarawagi et al, ${ }^{4}$ RPSD was draining into the cystic duct in only $0.8 \%$ of subjects.

It is confessed in this case that RPSD joining cystic duct was missed during gall bladder resection, and was identified and analysed retrospectively on MRCP, because its an extremely rare and un-expected finding. Our discussion faces the question: Is preoperative MRCP necessary for patients with gallstones, as the query was raised by Qiu, ${ }^{5}$ Rao, ${ }^{6}$ and their colleagues in their research studies with the same theme. This case highlights the importance of screening pre- operative MRCP before cholecystectomy as a mandatory investigation, not only to diagnose choledocholithiasis, but also to diagnose intra-hepatic and extra-hepatic bile duct complex variations, which, if known to the surgeon, can prevent excessive morbidity.

\section{CONFLICT OF INTEREST:}

Authors declared no conflict of interest.

\section{AUTHOR'S CONTRIBUTION:}

$\mathrm{MH}$ : Made substantial contributions to the design of the work; drafted the work and revised it critically for important intellectual content.

\section{REFERENCES}

1. Sahajpal AK, Chow SC, Dixon E, Greig PD, Gallinger S, Wei AC Bile duct injuries associated with laparoscopic cholecystectomy: Timing of repair and long-term outcomes. Arch Surg 2010; 145:757-63. 
2. Mortelé KJ, Ros PR. Anatomic variants of the biliary tree: MR cholangiographic findings and clinical applications. AJR Am J Roentgenol 2001; 177:389-94.

3. Sureka B, Bansal K, Patidar Y, Arora A. Magnetic resonance cholangiographic evaluation of intrahepatic and extrahepatic bile duct variations. Indian J Radiol Imaging 2016; 26:22-32.

4. Sarawagi R, Sundar S, Raghuvanshi S, Gupta SK, Jayaraman G. Common and uncommon anatomical variants of intrahepatic bile ducts in magnetic resonance cholangiopancreatography and its clinical implication. Pol J Radiol 2016; 81:250-5.

5. Yan Qiu, Zhengpeng Yang, Zhituo Li, Weihui Zhang, Dongbo Xue. Is preoperative MRCP necessary for patients with gallstones? An analysis of the factors related to missed diagnosis of choledocholithiasis by preoperative ultrasound. BMC Gastroenterol 2015; 15:158.

6. Rao GB, Nayak SR, Teja SBR, Palacharla R. Preoperative
MRCP: Is it necessary before routine laparoscopic cholecystectomy to exclude CBD stone-prospective study in tertiary care hospital. International surgery journal. Int Surg J 2017; 4:3633-37.

Mahnoor Hafeez

Dow Institute of Radiology, Dow University of Health Sciences (DUHS), Karachi, Pakistan

Correspondence to: Dr. Mahnoor Hafeez, Dow Institute of Radiology, Dow University of Health Sciences (DUHS),

Karachi, Pakistan

E-mail: mahnoor.hafeez@yahoo.com

Received: February 21, 2020; Revised: April 17, 2020;

Accepted: April 17, 2020

DOI: https://doi.org/10.29271/jcpsp.2020.05.553 